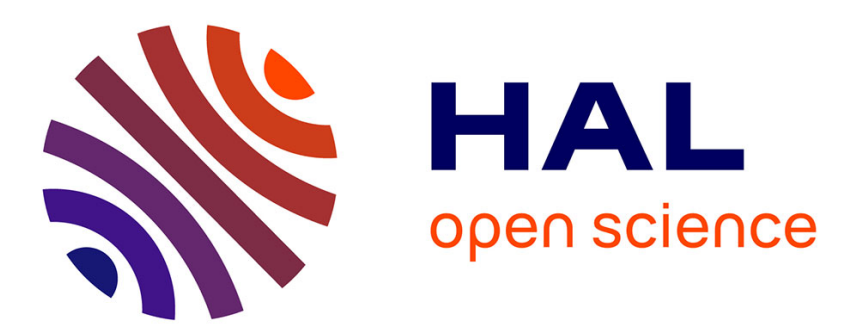

\title{
High resolution cw laser beam preamplified in a multipass cavity pumped by a CuHBr (HyBrID) laser
}

\author{
M. Laubscher, P. Segonds, J. P. Pique
}

\section{To cite this version:}

M. Laubscher, P. Segonds, J. P. Pique. High resolution cw laser beam preamplified in a multipass cavity pumped by a CuHBr (HyBrID) laser. Optics Communications, 2000, 184 (5-6), pp.425-435. 10.1016/S0030-4018(00)00918-4 . hal-01118344

\section{HAL Id: hal-01118344 \\ https://hal.science/hal-01118344}

Submitted on 3 Mar 2015

HAL is a multi-disciplinary open access archive for the deposit and dissemination of scientific research documents, whether they are published or not. The documents may come from teaching and research institutions in France or abroad, or from public or private research centers.
L'archive ouverte pluridisciplinaire HAL, est destinée au dépôt et à la diffusion de documents scientifiques de niveau recherche, publiés ou non, émanant des établissements d'enseignement et de recherche français ou étrangers, des laboratoires publics ou privés. 


\title{
High resolution cw laser beam preamplified in a multipass cavity pumped by a CuHBr (HyBrID) laser
}

\author{
M. Laubscher, P. Segonds and J.P. Pique \\ Laboratoire de Spectrométrie Physique (CNRS UMR 5588). \\ Université Joseph Fourier, BP. 87, 38402 St.Martin d'Hères, FRANCE
}

corresponding author :

Jean Paul Pique

Laboratoire de Spectrométrie Physique (CNRS UMR 5588)

Université Joseph Fourier, BP. 87, 38402 St.Martin d'Hères, FRANCE

Tel: $0476514745 \quad$ Fax: 0476514544

Email: jean-paul.pique@ spectro.ujf-grenoble.fr 


\begin{abstract}
The amplification of a cw high resolution (1 MHz) single-mode dye laser in a singlestage, multipass preamplifier based on a free flowing dye jet as amplifying medium is reported. The device is pumped by a homemade CuHBr-laser (HyBrID-laser) (18 $\mathrm{kHz}$ repetition rate, $30 \mathrm{~ns}$ pulse duration at half maximum and $80 \mathrm{~ns}$ at its base) and yields pulses of duration of $10 \mathrm{~ns}$ (at half maximum). In the studied pump energy domain the gain is a linear function of pump energy and the maximum value obtained so far with pump energy of $400 \mu \mathrm{J}$ is $25 \mu \mathrm{J}$ per pulse for an input beam power of 30 $\mathrm{mW}$. The corresponding gain is of the order of $10^{4}$. We believe that higher gain can be reach using better quality elements. The output beam is a high quality $\mathrm{TM}_{00}$ mode. The pulse shapes of the pump beam and the output beam are compared and a phenomenological model ("switched photon lifetime") is introduced that reproduces our observations with a good agreement.
\end{abstract}




\section{Introduction}

Tunable dye lasers are widely used in optical spectroscopy. Single-mode operation with low frequency jitter on the one hand and high output powers on the other, are obtainable with dye lasers, but, as a rule, not simultaneously. However, there is great interest in the production of single-frequency high-peak power pulses for multistep photoionization spectroscopy [1,2], isotope separation [3], LIDAR [4], laser beacon formation [5], medical application $[6,7] \ldots$

To generate high-quality tunable laser light, a cw dye laser or a pulsed dye laser oscillator are required to be pumped at relatively low power. Their low-power output must therefore be amplified in dye laser amplifiers to obtain a high-power laser pulse. The basic idea is therefore to produce a beam of high spectral quality and stability in a master laser, followed by a high-gain slave amplifier. Current amplifier configurations are mostly based on a series of dye cells, amplifying the seed beam in a multistage process [8]. A very efficient configuration based on a four-pass dye-cell amplifier has been demonstrated: $1.5 \mathrm{~mJ}$ at $5.6 \mathrm{~mJ}$ pumping energy at $10 \mathrm{~Hz}$ with 100 $\mathrm{mW}$ single-frequency $\mathrm{cw}$ beam has been obtained with a power gain of $2 \times 10^{6}$ [9]. However, low repetition rate, ASE (15\%) and poor beam quality made this configuration not suitable for our application. Without taking special precautions to increase the flow velocity of the dye solution near the cell walls, problems occur from the deposition of destructured dye molecules there on when using high pump energies and high repetition rates. To avoid these problems the use of a free dye jet has been suggested [10]. Inspired by confocal multipass amplifier cavities in the literature $[11,12]$, we have combined the dye jet technology with a confocal unstable cavity configuration to build a single-stage, multipass dye-jet amplifier. 
In this paper we report the amplification of a single-mode cw dye laser at 589 $\mathrm{nm}$ using this new amplifier configuration and a $\mathrm{CuHBr}$ pump laser. We begin by discussing in part 2 the concept of the amplifier configuration and its experimental realization. In part 3 we present the whole experimental setup used to create and to study high energy, frequency-stabilized laser pulses. The measurements obtained using this set-up are detailed in part 4 . We studied the gain and the efficiency of the device with respect to the different input parameters, as well as the spectral and temporal characteristics of the amplified beam. A simple theoretical description of the amplification process that reproduces our observations is presented in part 5 .

\section{Amplifier Configuration}

We report here a rapid description of the amplifier, which has been studied in detail in ref. [13] for modeless-laser application. Figure 1 is a schematic diagram illustrating the basic idea of the amplifier configuration used. The dye jet was located at the common focal point of a confocal unstable cavity that consisted of two lenses L and two mirror pairs $\mathrm{MP}_{1}$ and $\mathrm{MP}_{2}$. The mirror pair was a $90^{\circ}$ corner made of two plane mirrors with the purpose of producing an outgoing beam parallel and height shifted to the incoming beam. The two plane mirrors of one of the pairs $\left(\mathrm{MP}_{1}\right)$ were sufficiently separated in order to allow the input beam to enter the cavity. $\mathrm{MP}_{1}$ and $\mathrm{MP}_{2}$ are shifted one versus the other so as to produce a multipass cavity. The beam propagation schematic in figure 1 illustrates this principle. At each pass the beam was moving away from the optical axis of the cavity, but for each pass it was focused at the same point on the dye jet plane by the lenses L. The number of passes (typically 10 in our experiment) could be adjusted by translating a plane mirror $\mathrm{M}$ that served to outcouple the beam. The dye jet was pumped quasi-longitudinally. 
Due to chromatic aberrations introduced by the lenses $\mathrm{L}$ and in order to reduce reflection losses, spherical mirrors (SM) instead of the lenses have been used. The mirrors were symmetrically tilted by an angle $\alpha$ from the optical axis and thus formed a cavity in Z-form as depicted in figure 2. In order to reduce the creation of ASE we have introduced a dispersed element in the cavity, an equilateral prism in one of the arms of the Z-cavity, as will be discussed in more detail in section 4.1.

\section{Characterization using Gaussian optics}

The multipass geometry was directly inspired by a modeless laser cavity previously studied in our laboratory [13]. Its characteristics (neglecting the influence of the dye jet as being considered a plane parallel plate and not yet considering the prism) have been studied in detail using Gaussian ray propagation in the paraxial approximation. We recall here the main statements useful for the present discussion. Since the device was meant to be confocal in spite of the tilt of the spherical mirrors, it was used in a $4 \mathrm{f}$-configuration with $\mathrm{f}=\frac{R}{2 \cos (\alpha)}$ being the basic unit of distance between the mirror pairs and the spherical mirrors and between the spherical mirrors and the dye jet. $\mathrm{R}$ is the radius of curvature of the spherical mirrors $(313 \mathrm{~mm}$ in our setup). The tilt angle $\alpha$ was chosen to be $12.5^{\circ}$ in order to facilitate the placement of such elements as the outcoupling mirror and the disperse prism with the price of introducing a slight astigmatism to the laser beam. The result was a different focal length for the tangential and sagittal planes of the beam and thus a difference of the beam waists in those planes at the position of the dye jet. For the angle chosen this difference was inferior to 5\%. These parameters resulted in a value for $4 \mathrm{f}$ of 641.2 $\mathrm{mm}$, which gave a propagation time for one pass of $2.1 \mathrm{~ns}$. 
In order to profit of the $4 \mathrm{f}$-configuration it was necessary to form the beam waist of the input beam on a plane conjugated to the dye jet plane. The system had two such planes: those at $\mathrm{MP}_{1}$ and $\mathrm{MP}_{2}$. Therefore, the injection requirement to focus the beam to its waist on the dye jet for each pass was to place the input waist on the plane of $\mathrm{MP}_{1}$. The sizes of the waists on the mirror pairs (w1) and on the dye jet plane (w2) were linked by the following relations, for the tangential and sagittal planes respectively: $\quad w_{1}^{\tan g} w_{2}^{\tan g}=\frac{\lambda R}{2 \pi \cos (\alpha)} \quad$ and $\quad w_{1}^{\text {sagit }} w_{2}^{\text {sagit }}=\frac{\lambda R}{2 \pi} \cos (\alpha), \quad \lambda$ being the wavelength of the input beam.

In order to get enough gain and to avoid dye jet ablation we imposed a beam waist for the pump beam at the dye jet plane of $\mathrm{w}_{\mathrm{p}}=100 \mu \mathrm{m}$ (half-width). Matching $\mathrm{w}_{2}=\mathrm{w}_{\mathrm{p}}$ this imposed a beam waist $\mathrm{w}_{1}$ of $290 \mu \mathrm{m}$ at the plane mirror pairs, a condition that had to be realized by the input beam.

\section{Optics characteristics}

The diameter choices for the optics were mostly driven by the necessity to allow a sufficient number of passes through the amplifying medium. We have chosen standard dielectric coatings for all mirror surfaces (> 99\% reflectivity @ 590 nm / 0$45^{\circ}$ Melles Griot) and mounted every optical element on a XY translation stage. The plane mirrors forming the two mirror pairs MP were $25 \mathrm{~mm}$ by $8 \mathrm{~mm}$ rectangles, assembled at a $90^{\circ}$ angle, adjustable to a $0.1 \mathrm{mrad}$ precision. The spherical mirrors were held by a tip-tilt mount and had a diameter of $25 \mathrm{~mm}$. The mirror pairs MP were height-shifted by $1 \mathrm{~mm}$, which allowed a maximum of 10 passes through the structure without any spot overlap on any mirror.

Among the standard commercial prisms we have chosen a prism material of high dispersion (Flint). The equilateral prism was used in the position of minimal 
deviation in order maximize dispersion. Fortunately, this position corresponds to an incident angle close to Brewster's angle. Flint (F2) with a refractive index of 1.60 at $589 \mathrm{~nm}$ satisfied rather well this condition with a $4^{\circ}$ difference between position of maximum dispersion $\left(54^{\circ}\right)$ and Brewster's angle $\left(58^{\circ}\right)$.

\section{Dye jet characteristics}

The amplifying medium was a Rhodamine 6G dye in ethylene glycol $(n=1.43)$ with an emission range of approximately $80 \mathrm{~nm}$, centered at $590 \mathrm{~nm}$ when pumped by our CuHBr-laser. We used a concentration of $1.410^{-3} \mathrm{~mol} / \mathrm{l}$ in order to obtain only $15 \%$ transmission of the pump beam. To create the dye jet we used the sapphire nozzle of a Spectra Physics Ring Dye Laser (Model 380D) together with its dye circulator (Model 376B) and vibrating-accumulator (Model 372B). The circulator delivered approximately 6 bar average pressure which resulted in a jet speed of 35 $\mathrm{m} / \mathrm{s}$, enough to renew the amplifying medium between two successive pump laser pulses (18 kHz repetition rate). The nozzle was mounted on a XYZ translation stage, which allowed us to scan the jet surface for the best area and to adjust the jet position with respect to the common focal point of the spherical mirrors.

The jet thickness was measured to be $290 \mu \mathrm{m}$, using interference of light trapped inside the jet (mode spacing of light in a cavity formed by the jet walls) [13]. It was set vertically and could be turned to Brewster's angle $\left(55^{\circ}\right)$ in order to minimize reflection losses. However, turning the jet away from a position normal to the circulating laser beam increased the effective jet thickness and introduces aberrations due to a slightly inhomogeneous jet surface. We generally used an angle of $10^{\circ}$ in a compromise between beam quality and reflection losses. 


\section{Experimental Setup}

A schematic diagram of the complete experimental setup is shown in figure 3. It can be sub-divided into four parts: The commercial $\mathrm{cw}$ linear-oscillator (Coherent dye laser 599-21) pumped by a frequency-doubled Nd: YVO4 laser (Coherent Verdi), the multipass cavity (configuration as described in part 2), the pump laser (laboratorymade $\mathrm{CuHBr}$-laser) and the analyzing equipment (spectrometer, photodiodes...).

The tunable cw linear dye laser delivered a single-mode, frequency-stabilized ( $1 \mathrm{MHz}$ ), linear polarized beam of up to $100 \mathrm{~mW}$ average power with an output beam waist of $240 \mu \mathrm{m}$. It was used with a Rhodamine $6 \mathrm{G}$ solution in ethylene glycol and pumped by $2 \mathrm{~W} \mathrm{cw}$ power at $532 \mathrm{~nm}$, delivered by the doubled Nd-laser. The dye oscillator was followed by a double-pass Faraday optical isolator that suppressed back reflections that could interfere with the frequency stabilization. The isolator turned the polarization by $90^{\circ}$ to produce a beam polarized in the horizontal plane. This beam was then injected into the amplifier structure by way of the mirrors $M_{1}, M_{1}^{\prime}$ and $M^{\prime \prime}{ }_{1}$ and the lens $L_{1}\left(f_{1}=400 \mathrm{~mm}\right)$. The lens served to produce the input waist of $290 \mu \mathrm{m}$ at the entrance of the amplifier, as discussed in part 2.

The pump source used for the amplifier was a CuHBr-laser with a pulse repetition rate of $18 \mathrm{kHz}$. The laser has been thoroughly described in [14]. It was based on an unstable cavity and delivered, on average, pulse-trains that were made up of 4 peaks with a total pulse length at the base of $80 \mathrm{~ns}$ (30 ns at half maximum) and with energies of up to $3 \mathrm{~mJ}$ summed over two wavelengths: $510.6 \mathrm{~nm}$ and $578.2 \mathrm{~nm}$. The pulse shape of the $\mathrm{CuHBr}$ laser is reported in figures 8 -a, which will be discussed in more detail in section 4.3. The $510.6 \mathrm{~nm}$ line (green line) was selected using a dichroic beam splitter D, since it was better adapted to the R6G dye maximum absorption. The beam was guided by two mirrors $\mathrm{M}_{2}$ and $\mathrm{M}_{2}^{\prime}$ and focused on the dye 
jet plane by lens $L_{2}\left(f_{2}=250 \mathrm{~mm}\right)$ in order to obtain a beam waist of $\mathrm{w}_{1}=100 \mu \mathrm{m}$ (half width).

For spectral analysis we used either an Acton spectrometer whose spectral range is $100 \mathrm{~nm}$, or a Sopra spectrometer whose spectral range is $3.7 \mathrm{~nm}$. Spectra were always recorded using an 1100 pixels CCD camera (Princeton Instruments), with a theoretical resolution of $0.08 \mathrm{~nm}$ (Acton) and $0.003 \mathrm{~nm}$ (Sopra) and a dynamics of 16 bits. Limiting experimental resolutions, given by the optical transfer functions of the equipment, were $0.4 \mathrm{~nm}$ (Acton) and $0.01 \mathrm{~nm}$ (Sopra). Temporal measurements were made using Hamamatsu InGaAs photodiodes (G3476 series) whose rise time was of the order of $0.3 \mathrm{~ns}$ in connection with a $500 \mathrm{MHz}$-bandwidth digital oscilloscope (Tektronix TDS 640 A). Average powers were measured using Ophir powermeters.

\section{Experimental results}

In the following we will present the experimental results obtained with the setup as described in section 3. We were mainly concerned with measurements of gain and efficiency, spectral profiles and pulse shapes.

\section{$\underline{4.1 \text { Gain and efficiency }}$}

The main motivation for building the multipass amplifier was to achieve a high gain in a single-stage device with $\mathrm{nJ}$ input energy and about $10 \mu \mathrm{J}$ output energy pulse. High efficiency, which is not the requirement of this stage, would be obtained with additional cell-amplifiers. As the input energies are 4 orders of magnitude lower than the output energies, gain and efficiency take on the simplified form:

$$
\mathrm{g} \approx \mathrm{E}_{\text {out }} / \mathrm{E}_{\text {in }} \quad \eta \approx \mathrm{E}_{\text {out }} / \mathrm{E}_{\mathrm{p}}
$$


where $E_{\text {out }}, E_{\text {in }}$ and $E_{p}$ are respectively the energies per pulse of the outgoing, the incoming and the pump beams, respectively. The energies values $E_{\text {out }}$ and $E_{p}$ have been calculated based on the measured average powers and the repetition rate (18 $\mathrm{kHz}) . \mathrm{E}_{\mathrm{in}}$ is defined as the $\mathrm{cw}$ energy during a time corresponding to the base of the pump pulse (80 ns).

Figure 4 shows the dependence of output energy as a function of input energy for different pump situations. For output energies below approximately $10 \mu \mathrm{J}$ we observe a linear dependence, with different slopes depending on the pump energy. For the two measurement series at $E_{p}>200 \mu \mathrm{J}$, output energies surpass this limit and a saturation regime sets in. Saturation is attained for lower input energies as $E_{p}$ increases. In order to maximize efficiency it is preferable to avoid the saturation regime. In subsequent measurements we therefore fixed the input energy to $2.4 \mathrm{~nJ}$ ( $30 \mathrm{~mW}$ of $\mathrm{cw}$ laser). The gains that can be deduced from the shown measurements are of the order of $10^{4}$.

Figure 5-a reports the experimental energy per pulse of the output beam as a function of pump energy. In the pump-energy domain that our equipment permitted us to study, we find a linear relationship between output power and pump power with a slope-efficiency of $9 \%$ and an amplifying threshold at about $50 \mu \mathrm{J}$. The gain of the amplifier increased with pump energy, whereas the efficiency stayed constant in the regime studied. In order to optimize the device in gain as well as efficiency it would be necessary to determine the saturation regime for the pump energy. It is well known that the gain will eventually saturate for higher pump energies, but at this time we cannot yet give the saturation value. Preliminary experiments have shown that the linear behavior still remains for pump energies of up to $2 \mathrm{~mJ}$. The upper limit of the device is determined by the stability of the free jet at high pump energies. Thermal 
effects deteriorate the optical qualities of the dye jet and introduce strong aberrations. Dye jet ablation occurs for still higher pump energies.

\subsection{Spectral characteristics}

One main difficulty in amplifying a laser beam was the suppression of amplified spontaneous emission (ASE), which causes background noise and reduces the gain of the system. Our approach to minimize ASE was to introduce a prism as disperse element in the cavity, as mentioned in part 2. By spatially separating the wavelength components of the laser beam, the pumped region of the dye jet played the role of a spatial filter. Figure 6 shows the result of a ray trace calculation indicating the filtered spectral window using three standard prism materials (BK7, F2 and SF11) for a size of the pumped dye jet region of $200 \mu \mathrm{m}$ in diameter $\left(\mathrm{w}_{1}=\mathrm{w}_{\mathrm{pump}}=100 \mu \mathrm{m}\right)$. Spectrometer measurements underline the effect of the prism. Figure 7-a shows the spectrum of the amplified beam with and without prism using the Acton spectrometer. As the resolution is instrument-limited, we used the Sopra spectrometer (figure 7-b) for higher resolution measurements. However, no ASE is measurable with our equipment (the dynamics and the read-noise of the CCD camera are respectively $2^{16}$ and $5 \mathrm{rms})$.

\subsection{Temporal profile}

In figures 8 -a we show the pulse shape of the output beam leaving the multipass amplifier. It is compared to the pulse shape of the pump beam. Both pulses were recorded using two simultaneously triggered photodiodes and averaged over 200 pulses. Figure 8-a depicts the situation where the dye jet is oriented at $10^{\circ}$ from the optical axis (which implies reflection losses). The pulse width of the output beam is $10 \mathrm{~ns}$ at half maximum A time shift of about $8 \mathrm{~ns}$ corresponds to the response time of 
the device due to the multipass amplification process. The interpretation of this figure will be discussed in detail in the next paragraph.

\section{5. "Switched photon lifetime" model}

The experimental conditions studied here are not trivial to describe theoretically. All intervening characteristic times are of the same order of magnitude, which makes a steady state approach impracticable. The time between consecutive passes of the laser beam through the amplifying medium was $2.1 \mathrm{~ns}$, the lifetime of the excited dye molecule was of the order of $4 \mathrm{~ns}$, the total time of light propagation between the first and last passages through the dye jet was about $19 \mathrm{~ns}$ and the pump laser pulses had a duration of $80 \mathrm{~ns}$ (at its base) with a characteristic structure that varied considerably of the order of $20 \mathrm{~ns}$.

An exact theoretical description of the system is difficult. We presented here is a simple phenomenological model that is found to be physically satisfying and that can reproduce our experimental observations. Considering the multipass amplifier as a laser cavity with varying losses in time. Losses are considered minimal (mainly determined by mirrors and jet) during the propagation time of an incoming beam through the multipass geometry (19 ns), but they raise sharply once the front end of the amplified pulse reaches the outcoupling mirror (similar in a Q-Switched cavity). Losses are expressed using the notion of the photon lifetime in the cavity which is strongly time dependent.

\section{$\underline{\text { Rate equations }}$}

Following the example of numerous authors we consider the dye molecule as a 4level-system, which results in a set of four coupled rate equations [15]: 


$$
\begin{aligned}
& \frac{d N_{0}}{d t}=\frac{N_{1}}{\tau_{10}}-I_{p} \sigma_{p}\left(N_{0}-N_{3}\right) \\
& \frac{d N_{1}}{d t}=-\frac{N_{1}}{\tau_{10}}+\frac{N_{2}}{\tau_{21}}+I \sigma_{l}\left(N_{2}-N_{1}\right) \\
& \frac{d N_{2}}{d t}=\frac{N_{3}}{\tau_{32}}-\frac{N_{2}}{\tau_{21}}+I \sigma_{l}\left(N_{2}-N_{1}\right) \\
& \frac{d N_{3}}{d t}=-\frac{N_{3}}{\tau_{32}}+I_{p} \sigma_{p}\left(N_{0}-N_{3}\right)
\end{aligned}
$$

The situation is illustrated in figure $9 . N_{i}$ is the population intensities of level $i, \tau_{i j}$ the lifetime of the transition i to $\mathrm{j}, \sigma_{1}$ and $\sigma_{\mathrm{p}}$ emission and absorption cross sections for the amplified beam and the pump laser, respectively. $I_{p}$ is the intensity of the pump beam and I that of the amplified beam.

Given that the transitions 3 to 2 and 1 to 0 are rapid, non-radiative transitions ( $\tau_{32}$ and $\tau_{10}$ are of the order of picoseconds) compared to $\tau_{21}$ (3.7 ns after [15]), it was possible to make the following simplifications:

$$
\begin{aligned}
& \frac{d N_{3}}{d t}=\frac{d N_{1}}{d t}=0 \\
& N_{1}<<N_{2} \\
& N_{3}<<N_{0}
\end{aligned}
$$

This leads to the reduction to an effective two-level system, described by: 


$$
\begin{aligned}
& \frac{d N_{0}}{d t}=\frac{N_{2}}{\tau_{21}}-I_{p} \sigma_{p} N_{0}+I \sigma_{l} N_{2} \\
& \frac{d N_{2}}{d t}=-\frac{d N_{0}}{d t}
\end{aligned}
$$

The intensity evolution of the amplified beam is given by:

$$
\frac{d I}{d t}=c \frac{l}{L} \sigma_{l} N_{2} I-\frac{I}{T}
$$

where $1 / \mathrm{L}$ is the filling factor of the dye medium (thickness 1 ) inside the cavity (length $\mathrm{L})$ and $\mathrm{T}$ the photon lifetime in the cavity.

\section{$\underline{\text { Numerical resolution }}$}

For the numerical resolution (see table I) the characteristic dye values from [16] were chosen, an analytical fit of the measured pump pulse shape (figure 8-a) and the values $1(290 \mu \mathrm{m})$ and $\mathrm{L}(641 \mathrm{~mm})$ for our setup. Furthermore, an injected beam of $30 \mathrm{~mW}$

power and an effective pump energy $E_{p}^{\text {eff }}$ of $150 \mu \mathrm{J}$ was assumed. This value will be discussed later on.

$\mathrm{T}$ is the key of our "switched photon life time" model and the only fitting function. As indicated above its value was varied in a smoothed-step-like manner from a maximum (low losses) to a minimum value (open cavity) depending on time. To do this, an analytical form using a tanh-function was used:

$T(t)=\frac{T_{\max }}{2}\left[1-\tanh \left(a\left(t-T_{s}\right)\right]+T_{\min }\right.$

where $T_{\max }$ and $T_{\min }$ are the maximum and minimum values of $T$, respectively, $T_{s}$ the "switching time" where the regime changes and a the constant that determines the steepness of the step-function. The initial value $\mathrm{T}_{\max }$ was evaluated using the 
indicated mirror coefficients in part 2 and Fresnel equations for reflections on the dye jet and the prism. Realistic values for our setup are 30-40 $\mathrm{ns}$. Considering $\mathrm{T}_{\min }$ to be the minimum time a created photon stays in the amplifier structure before leaving it. This time is less or equal to the propagation time for one passage in the multipass amplifier, i.e. $2.1 \mathrm{~ns} . \mathrm{T}_{\mathrm{s}}$ should be of the order of $n . T_{\mathrm{r}}$, with $\mathrm{n}$ the number of passes (10) and $\mathrm{T}_{\mathrm{r}}$ the one pass time (2.1 ns).

A fitting procedure resolving the set of differential equations using these fixed and variable parameters, gives the optimum theoretical amplified laser pulse shown in figure 8-b. For reference the analytical form of the pump pulse used for this calculation is superimposed. Comparing the theoretical figure 8-b to the experimental one 8 -a, it can be seen that our "switched photon lifetime" model reproduces quite well the observed pulse shapes. As for the absolute energy values of the resulting amplified laser pulses, we can fit the function $\mathrm{T}(\mathrm{t})$ in reasonable limits to find output energies of about $30 \mu \mathrm{J}$. The delay between pump and output pulses is also in good agreement. The fitted switching-time function is represented on figure 10 .

We would like now to clarify the notion and the value of the effective pump energy. $\mathrm{E}_{\mathrm{p}}{ }^{\text {eff }}$ is the pump energy, which participates in the amplification process. Its value is 2.7 smaller than the experimental pump energy. This difference is interpreted in the cumulative pump losses: transmission through the jet $(\sim 15 \%)$, reflection and diffusion on the jet faces $(>10 \%)$, none-polarized pump beam, imperfect pump and cavity beam waists overlap, none-radiative losses (triplet...) and so on.

The "switched photon lifetime" model reproduces also well the output energy versus pump energy. Figure 5-b shows that if we take the 2.7 factor between the effective and experimental pump energy into account, the slope-efficiency and threshold are in very good agreement. It is important to note that if the experimental 
efficiency is only $6 \%\left(\mathrm{E}_{\text {out }} / \mathrm{E}_{\mathrm{p}}\right)$, the effective slope-efficiency is $20 \%$. The effective slope-efficiency is defined as $2.7 \cdot E_{\text {out }} /\left(E_{p}-E_{s}\right)$, where $E_{s}$ is the pump energy threshold.

\section{Conclusion}

We have achieved gains of the order of $10^{4}$ at a repetition rate of $18 \mathrm{kHz}$ in a single-stage laser amplifier using a free dye jet. The working wavelength was 589 $\mathrm{nm}$, stabilized to within $1 \mathrm{MHz}$. Using a CuHBr pump laser with a pulse duration of approximately $30 \mathrm{~ns}$ (at half maximum), an energy of up to $400 \mu \mathrm{J}$ per pulse and an input energy of $2.4 \mathrm{~nJ}$. We obtained an output energy of $25 \mu \mathrm{J}$ and a pulse duration of approximately $10 \mathrm{~ns}$ (at half maximum). For the range of pump energies studied, the gain is a linear function of the pump energy and the energy conversion efficiency is about $6 \%$ with an effective slope-efficiency of $20 \%$. But the goal of our preamplifier was to reach enough energy in order to use additional high efficient amplifier stages. Preliminary experiment has shown that mean power in the range 10-100 watts can be obtained.

Using a dispersed equilateral prism inside the amplifier system, the amplified spontaneous emission could be reduced under the detection limit of our equipment.

A "switched photon lifetime" model was introduced that reproduced the observed pulse shapes, delay, energy, efficiency and threshold values. The results we present here have shown a dramatic reduction of the pulse width (30 ns $\rightarrow 10 \mathrm{~ns}$ ), which is due to nonlinear effects during the multipass amplification process. This reduction is a problem for high efficient next amplifier stages. Believing in our model we found that in order to extract an output pulse of the same width as the pump we

must reduce the number of passes and increases $E_{p}{ }^{e f f}$. Figure 11 shows that with 6 passes and an effective pump energy of $400 \mu \mathrm{J}$ the output pulse follows the pump 
pulse. Higher effective pump energy can be obtained either with lower pump losses or increasing pump power.

\section{Acknowledgements}

The CNRS (ULTIMATECH) and the MENRT (DS2) have supported this work. The authors thank A. Katchanov and F. Stoeckel for useful discussions. 


\section{References}

[1] R.V.Ambartzumian and V.S.Letokhow, Appl.Opt. 11, (1972) 354-358.

[2] V.S.Letokhow, Laser Photoionization Spectroscopy (Academic, New York, 1987).

[3] P.T.Greenland, Contemp. Phys. 31, (1990) 405-424.

[4] E.Frejafon, J.Kasparian, P.Rambaldi, B.Vezin, V.Boutou, J.Yu, M.Ulbricht, D.Weidauer, B.Ottobrini, E.deSaeger, B.Kramer, T.Leisner, P.Rairoux, L.Woste, J.P .Wolf, Europ. Phys. J. D 4, No.2, (1998) 231-8.

[5] R.Foy, A.Migus, F.Biraben, G.Grynberg, P.R.McCullough, M.Tallon, Astron. Astrophys. Suppl. Ser. 111, (1995) 569-578.

[6] C.E. Webb, High-Power Dye Lasers, F.J.Duarte, ed. (Springer-Verlag, Berlin, 1991); Chap.5 , pp.143-182.

[7] L.Goldman, Dye Laser Principles, F.J.Darte and L.W.Hillman, eds. (Academic, New York, 1990), Chap.10, pp.419-432.

[8] S.Lavi, G.Bialolanker, M.Amit, D.Belker, G.Erez, E.Miron, Opt.Comm. 60, number 5, (1986) 309.

[9] E.S. Lee and J.W. Hahn, Optics Letters, 21, number 22, (1996) 1836.

[10] T.S. Petrov, N.V. Sabotinov, S.T. Trendafilov, Opt. Comm. 84, number 5,6, (1991) 295.

[11] P.Georges, F.Estable, F.Salin, J.P.Poizat, P.Gangier, A.Brun, Opt.Lett. 16, number 3, (1991) 144.

[12]R.L.Fork, F.A.Beisser, D.K.Fork, Revue Phys. Appl. 22, (1987) 1665.

[13] D. Müller, Diplomarbeit, Université Joseph Fourier, Grenoble (1998).

C.D’Orgeville, D.Müller, A. Katchanov and J.P.Pique, to be published. 
[14] P.Coutance, G.Naylor, J.P.Pique, IEEE J. Quantum Electron. 31, (1995) 10.

[15] F.J. Duarte, L.W. Hillman (editors), Dye laser principles, Academic Press 1990.

[16] B.B. Snavely, Proc. IEEE 57, (1969) 1374. 


\section{Table caption}

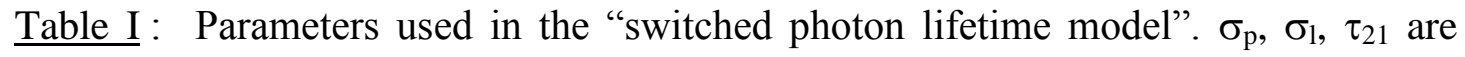
fixed values (after [15]). $\mathrm{N}_{0}$ value is calculated using the dye concentration (see text). $\mathrm{I}(\mathrm{t}=0)$ corresponds to an initial injected power of $30 \mathrm{~mW}$ with a $200 \mu \mathrm{m}$ beam waist. $\mathrm{T}_{\max }, \mathrm{T}_{\min }$ and $\mathrm{T}_{\mathrm{s}}$ are the only fitted parameters. 
Table I

\begin{tabular}{|l|l|}
\hline Parameter & \multicolumn{1}{c|}{ Value } \\
\hline$\sigma_{\mathrm{p}}\left(\mathrm{cm}^{2}\right)$ & $1.34 \cdot 10^{-16} \mathrm{~cm}^{2}$ \\
\hline$\sigma_{\mathrm{l}}$ & $0.6 \cdot 10^{-16} \mathrm{~cm}^{2}$ \\
\hline$\tau_{21}$ & $3.7 \mathrm{~ns}$ \\
\hline $\mathrm{N}_{0}(\mathrm{t}=0)$ & $0.9 \cdot 10^{18}$ photons $/ \mathrm{ns} / \mathrm{cm}^{2}$ \\
\hline $\mathrm{N}_{2}(\mathrm{t}=0)$ & 0 \\
\hline $\mathrm{I}(\mathrm{t}=0)$ & $1.87 \cdot 10^{12}$ photons $/ \mathrm{ns} / \mathrm{cm}^{2}$ \\
\hline $\mathrm{T}_{\max }$ & $31 \mathrm{~ns}$ \\
\hline $\mathrm{T}_{\min }$ & $1.3 \mathrm{~ns}$ \\
\hline $\mathrm{T}_{\mathrm{s}}$ & $17 \mathrm{~ns}$ \\
\hline $\mathrm{a}$ & 0.4 \\
\hline $\mathrm{E}_{\mathrm{p}}^{\text {eff }}$ & $150 \mu \mathrm{J}$ \\
\hline
\end{tabular}




\section{Figure captions}

Figure 1: Basic concept of the multipass amplifier.

Figure 2: Configuration of our multipass amplifier (top view).

Figure 3: Schematic diagram of the experimental setup. D stands for dichroic beam splitter and F.I. for Faraday isolator.

Figure 4: Energy per pulse of the output beam measured as a function of the energy of the input beam for different pump energies. The intra-cavity prism was not present in the experiment.

Figure 5: a) experimental pulse energy dependence between the output beam and the pump beam for an input energy of $2.4 \mathrm{~nJ}, \mathrm{~b}$ ) theoretical corresponding curve using the "switched photon lifetime" model.

Figure 6: Single pass spatial separation of the different wavelengths of the amplified beam in the dye jet plane induced by prisms made of BK7 (solid line), F2 (dotted line) and SF11 (dashed line). The pumped region of the jet has a diameter of $200 \mu \mathrm{m}$.

Figure 7: a) spectrum of the output beam with and without a prism in the multipass amplifier, using the Acton spectrometer. b) Spectrum with prism using the higher resolution Sopra spectrometer. Line widths are instrument limited.

Figure 8: a) experimental recorded pulses shape of the CuHBr pump laser (solid line) and amplifier output beam (dotted line). Recorded pulse shapes were averaged over 200 pulses for the dye jet oriented at $10^{\circ}$ from the optical axis and taken with two simultaneously triggered photodiodes in order to measure any time delay. b) Corresponding fitted pulses using the "switched photon lifetime" model. Effective pump energy $E_{\text {eff }}$ of $150 \mu \mathrm{J}$ has been used (see text and table I).

Figure 9: Schematic diagram of a 4-level energy system.

Figure 10: Time dependence of the intra-cavity photon lifetime. 
Figure 11: Output pulse simulation using the "switched photon lifetime" model with 6 passes and $\mathrm{E}_{\mathrm{p}}{ }^{\mathrm{eff}}=400 \mu \mathrm{J}$. 
Figure 1

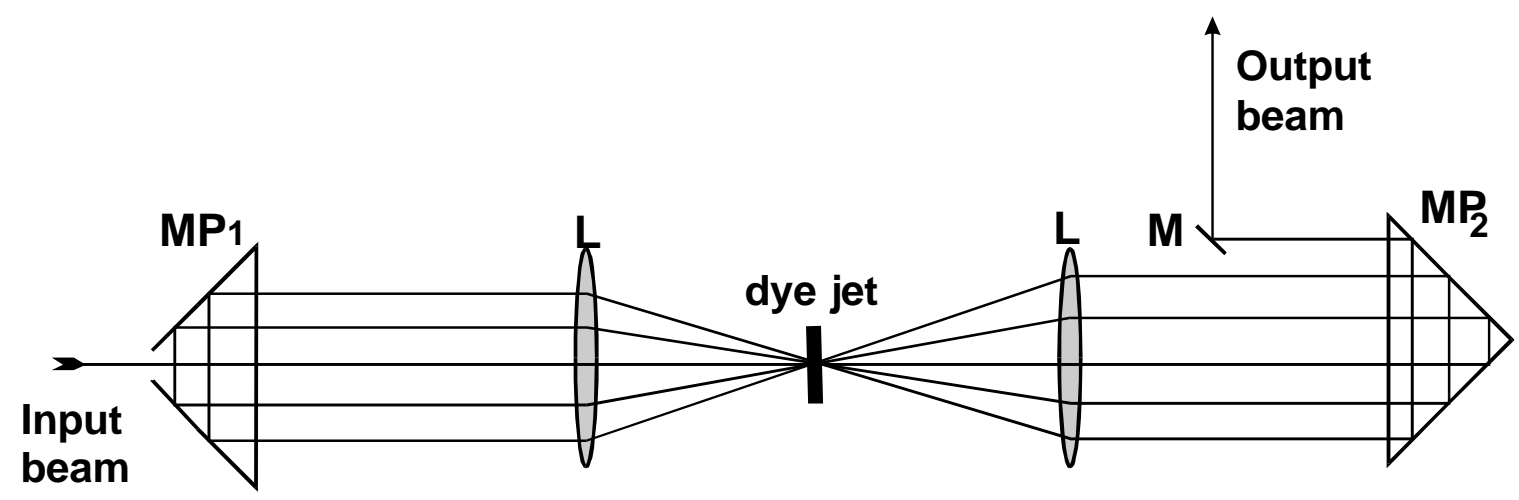


Figure 2

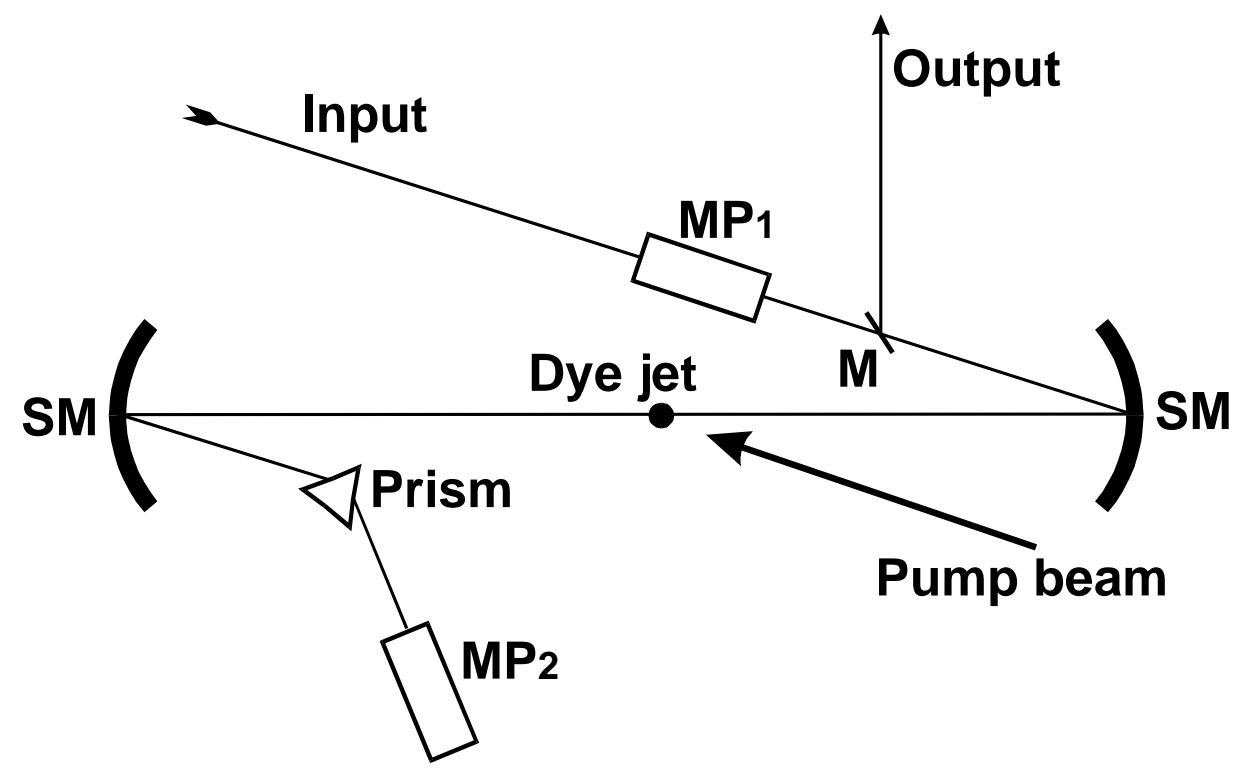


Figure 3

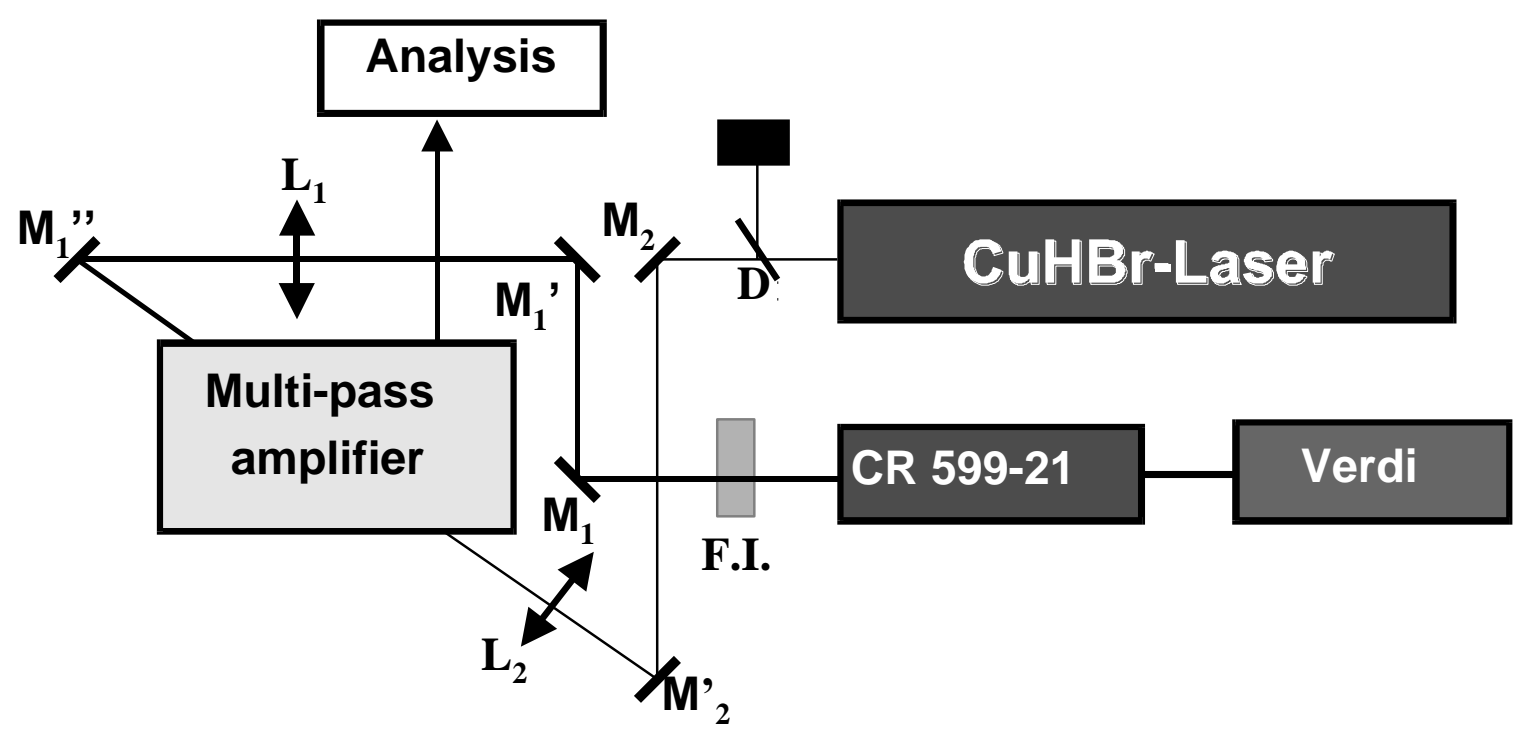


Figure 4

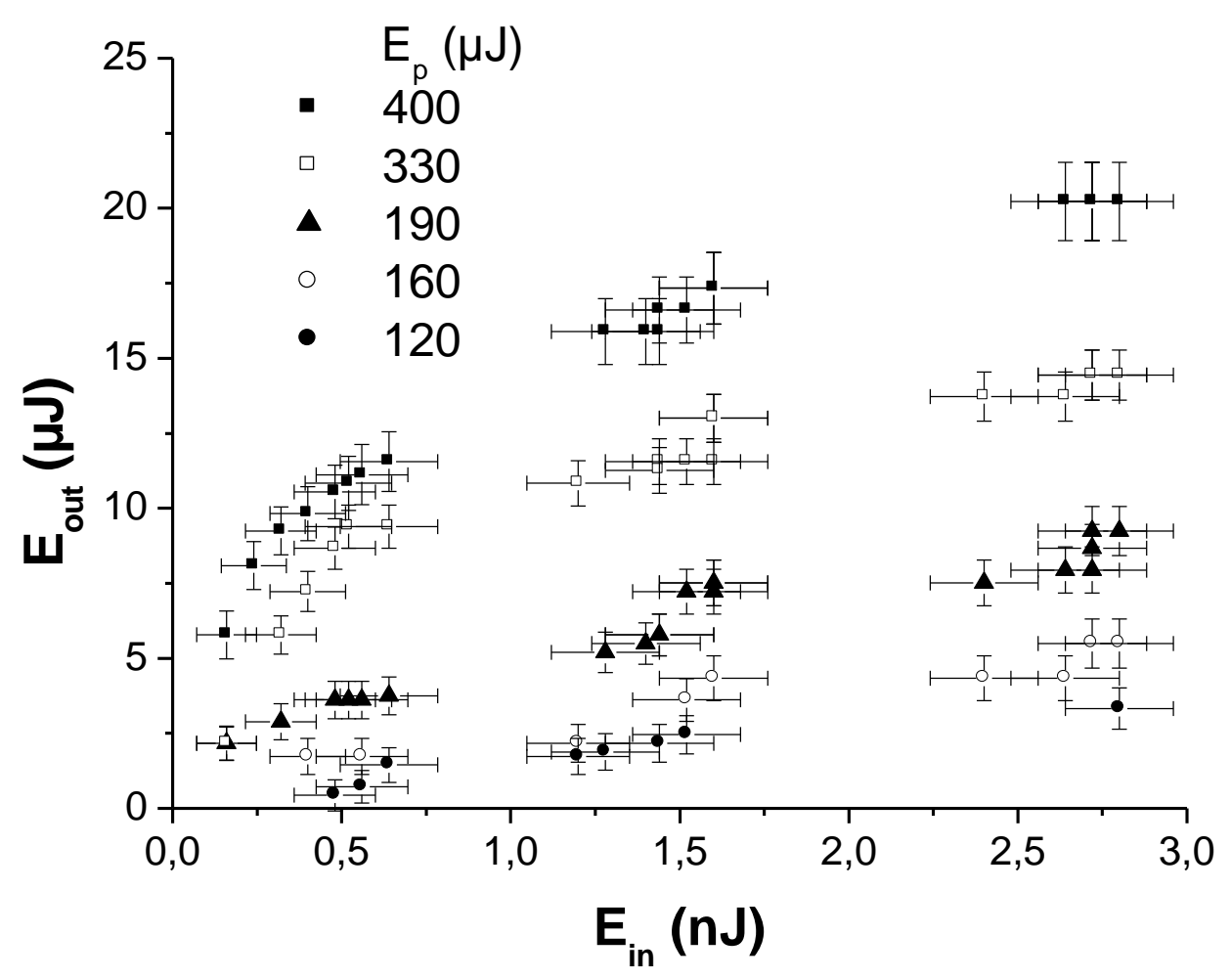


Figure 5-a

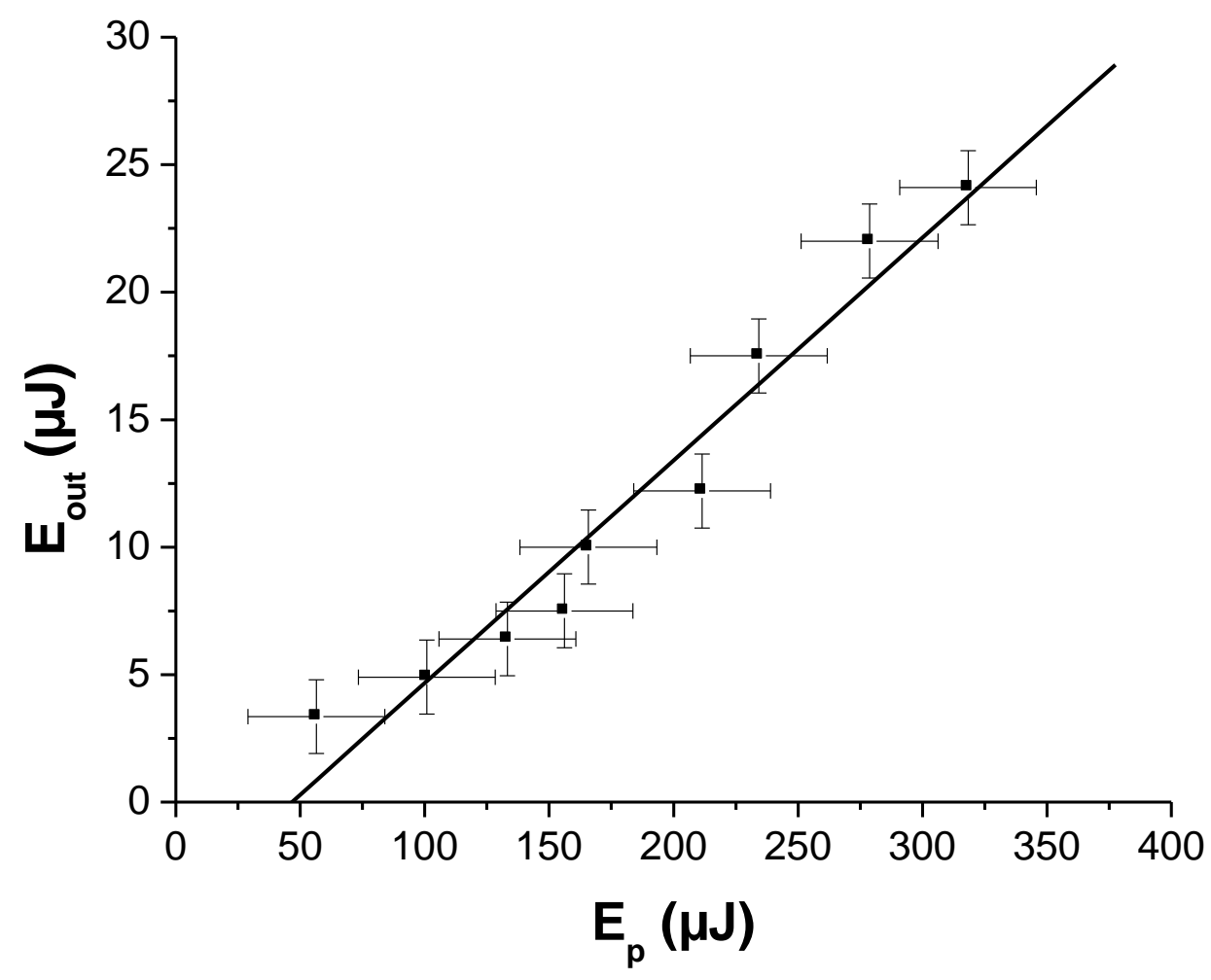

Figure 5-b

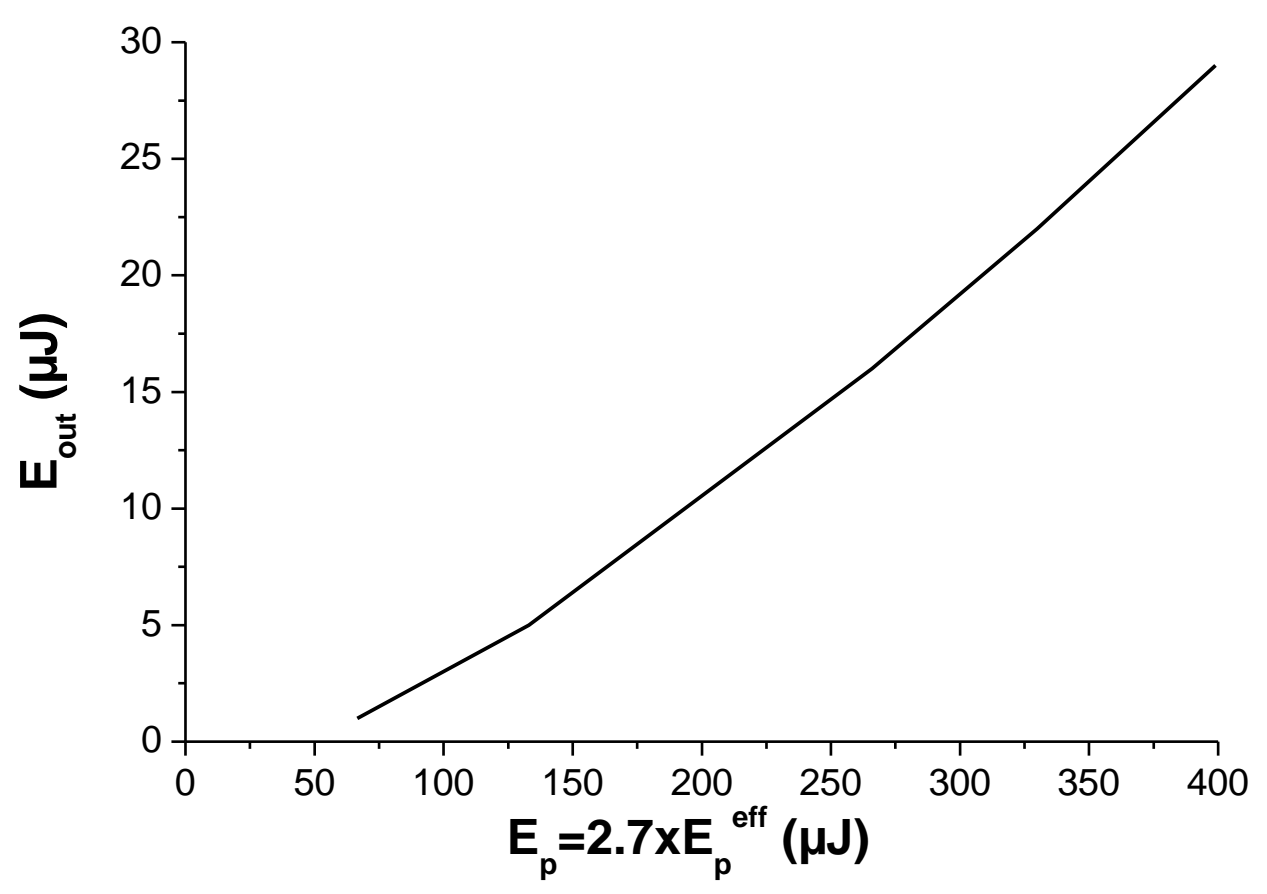


Figure 6

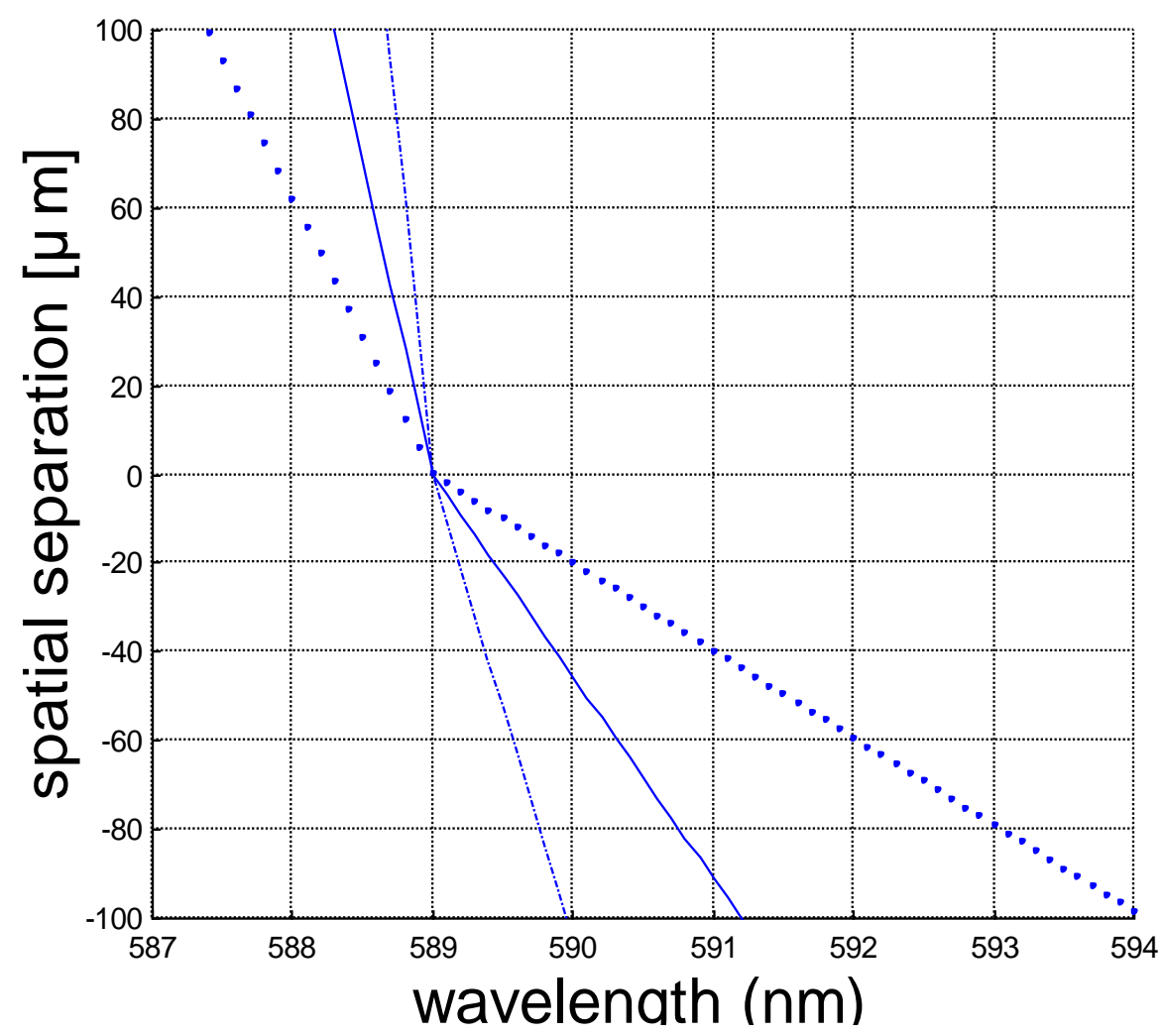


Figure 7-a

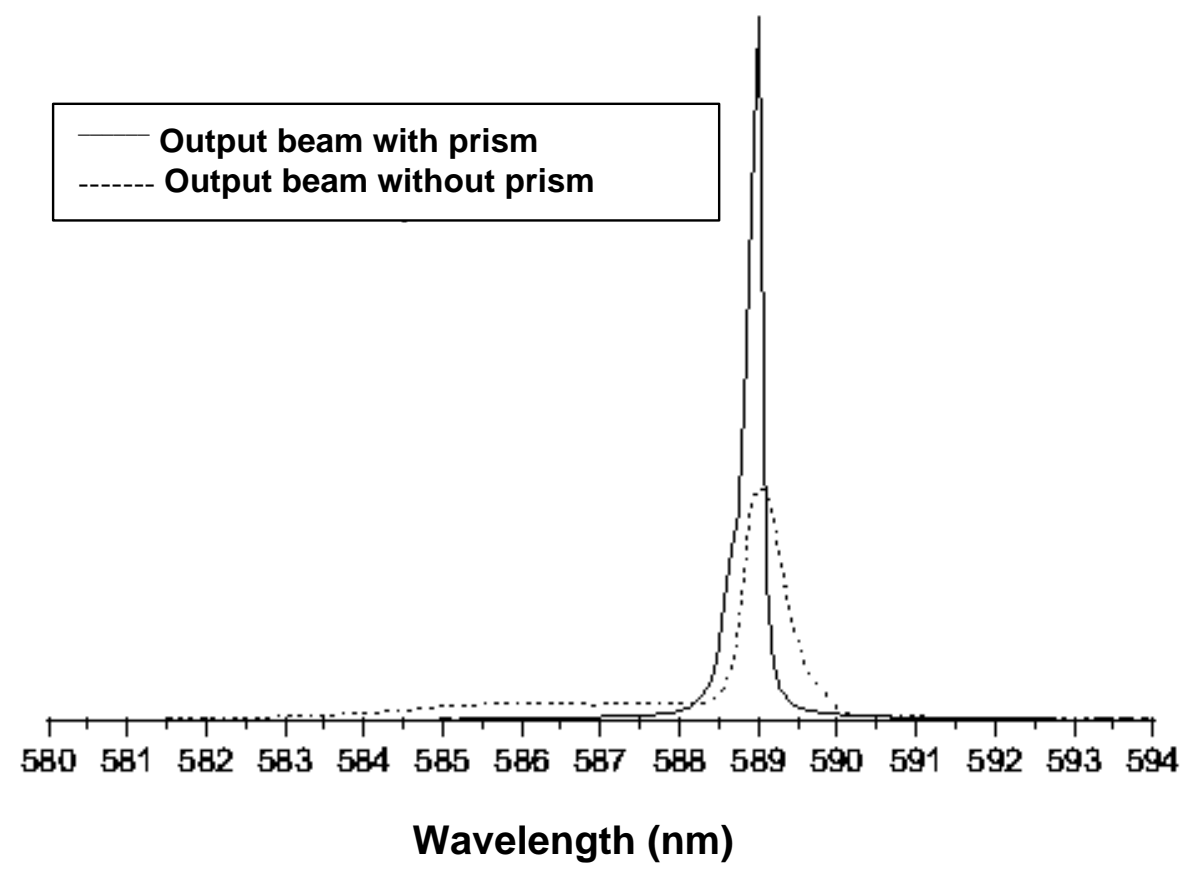


Figure 7-b

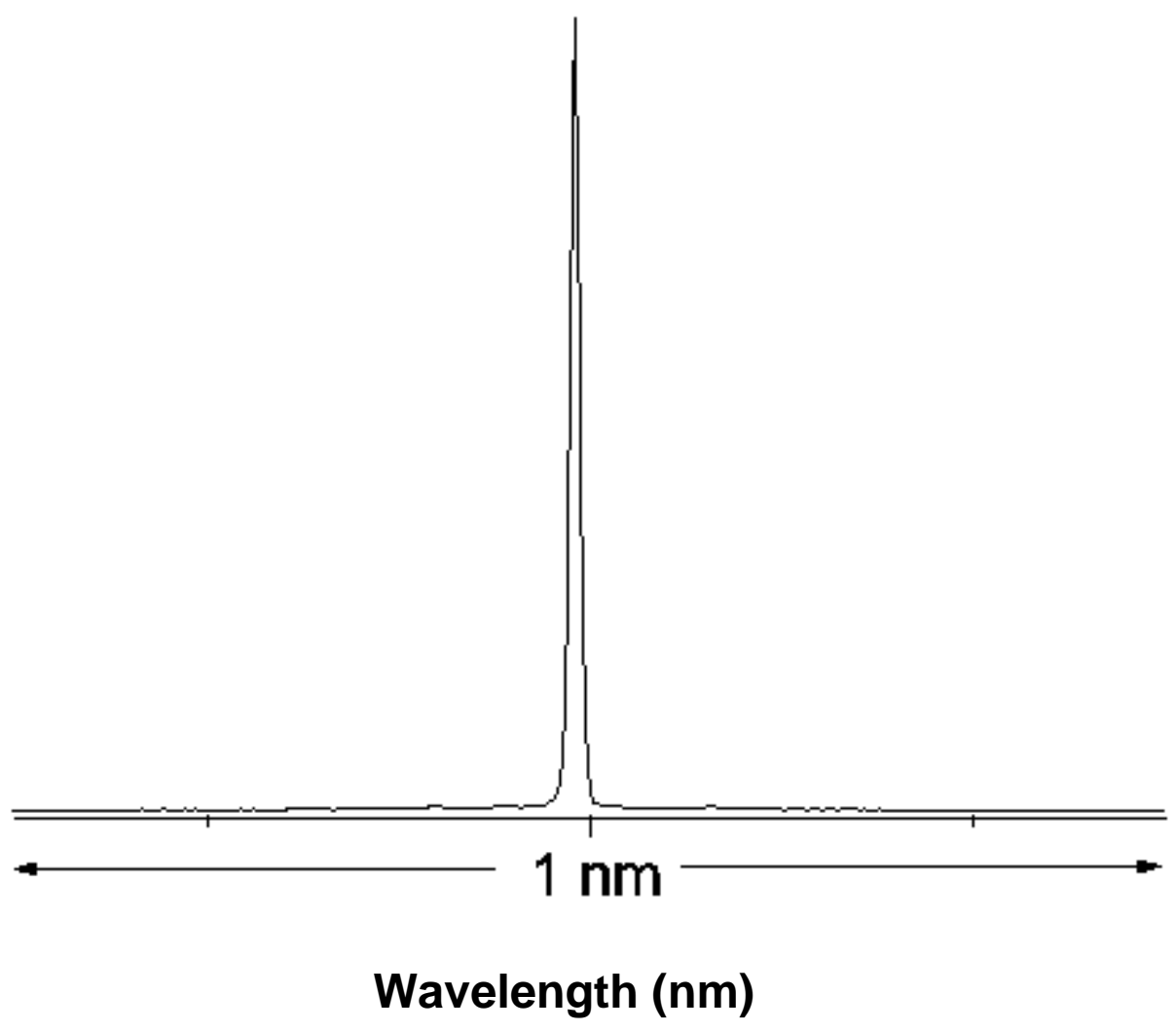


Figure 8-a

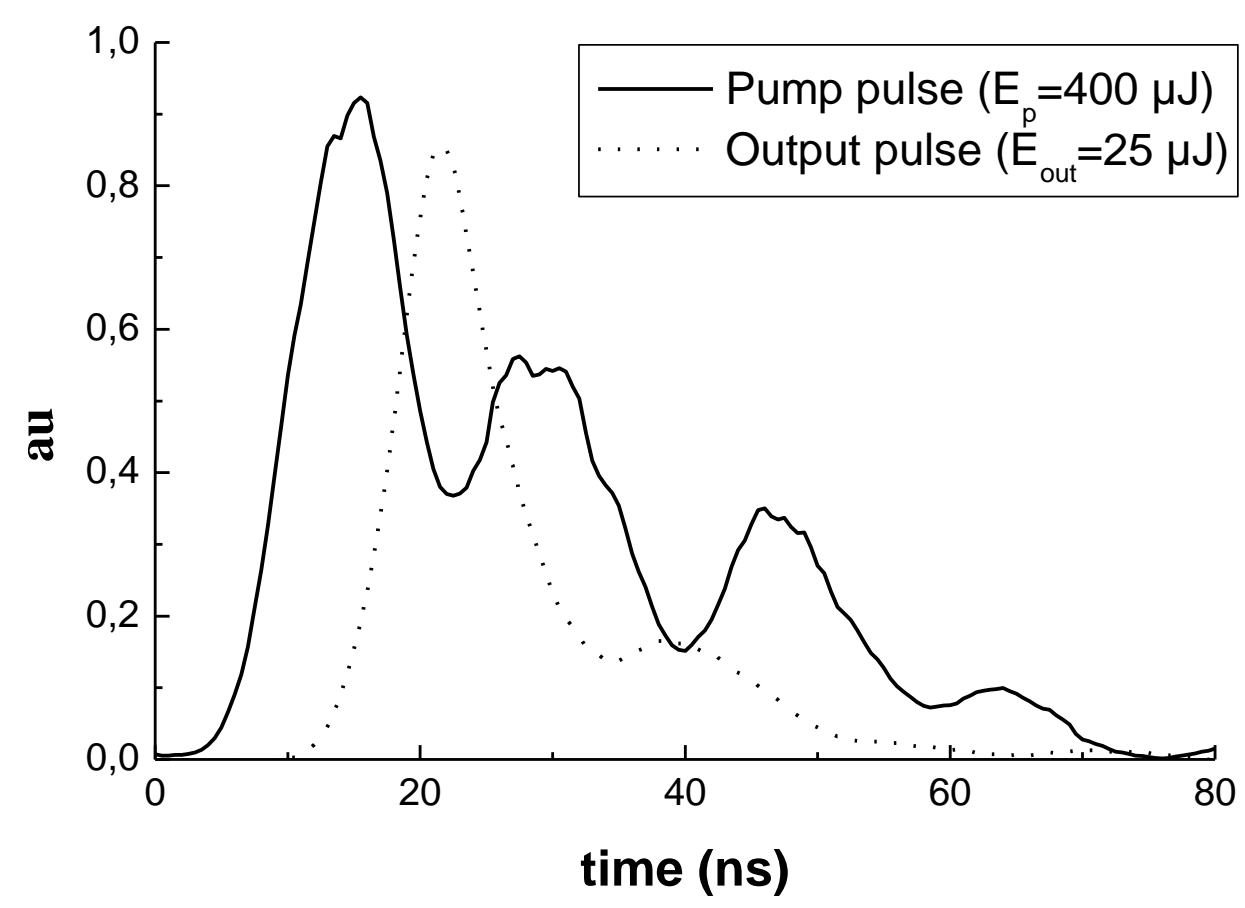

Figure 8-b

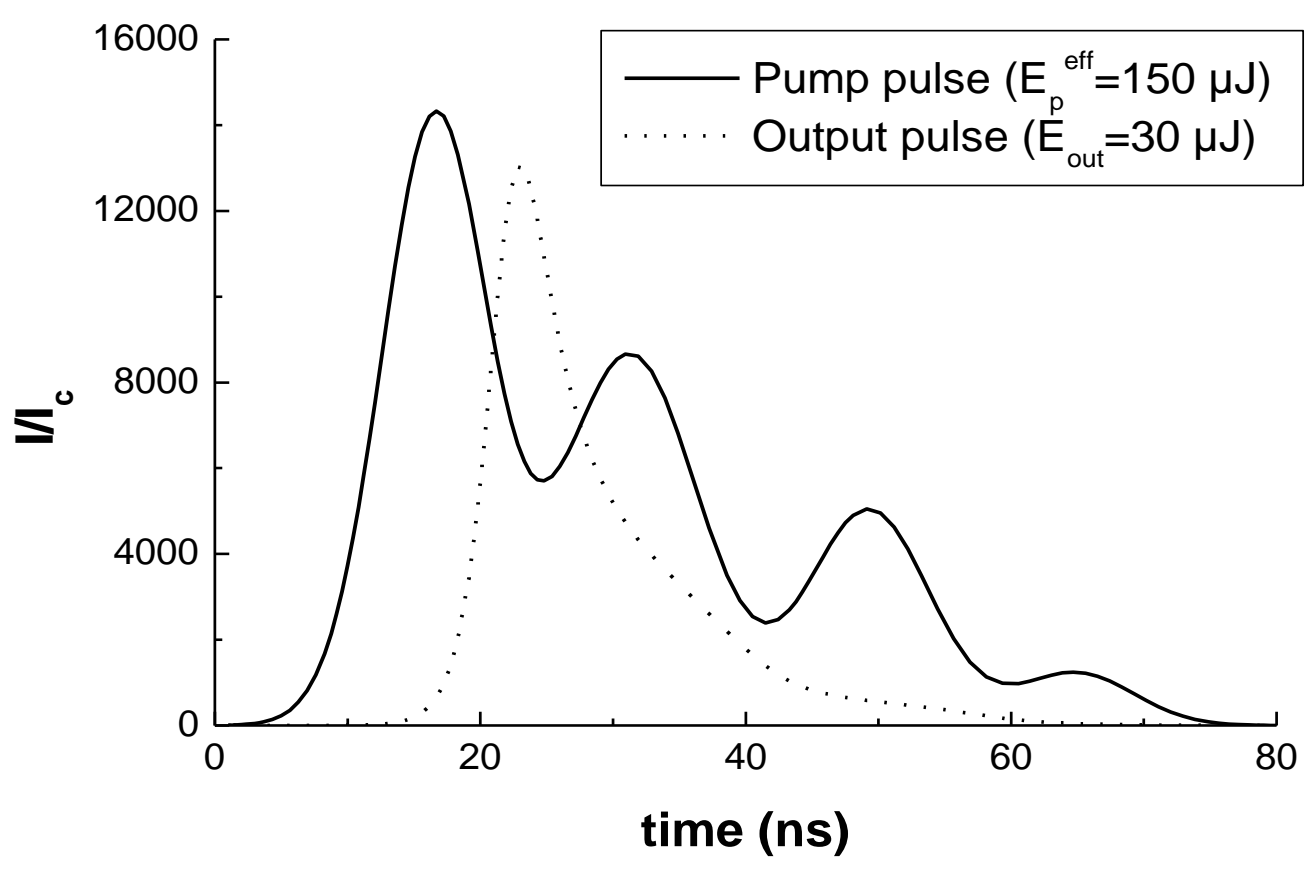


Figure 9

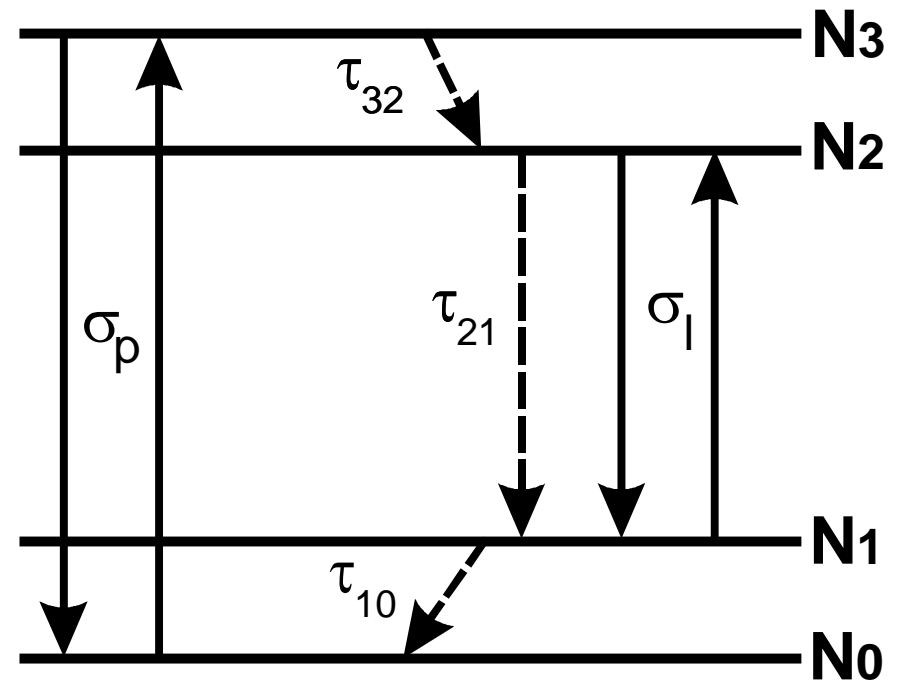


Figure 10

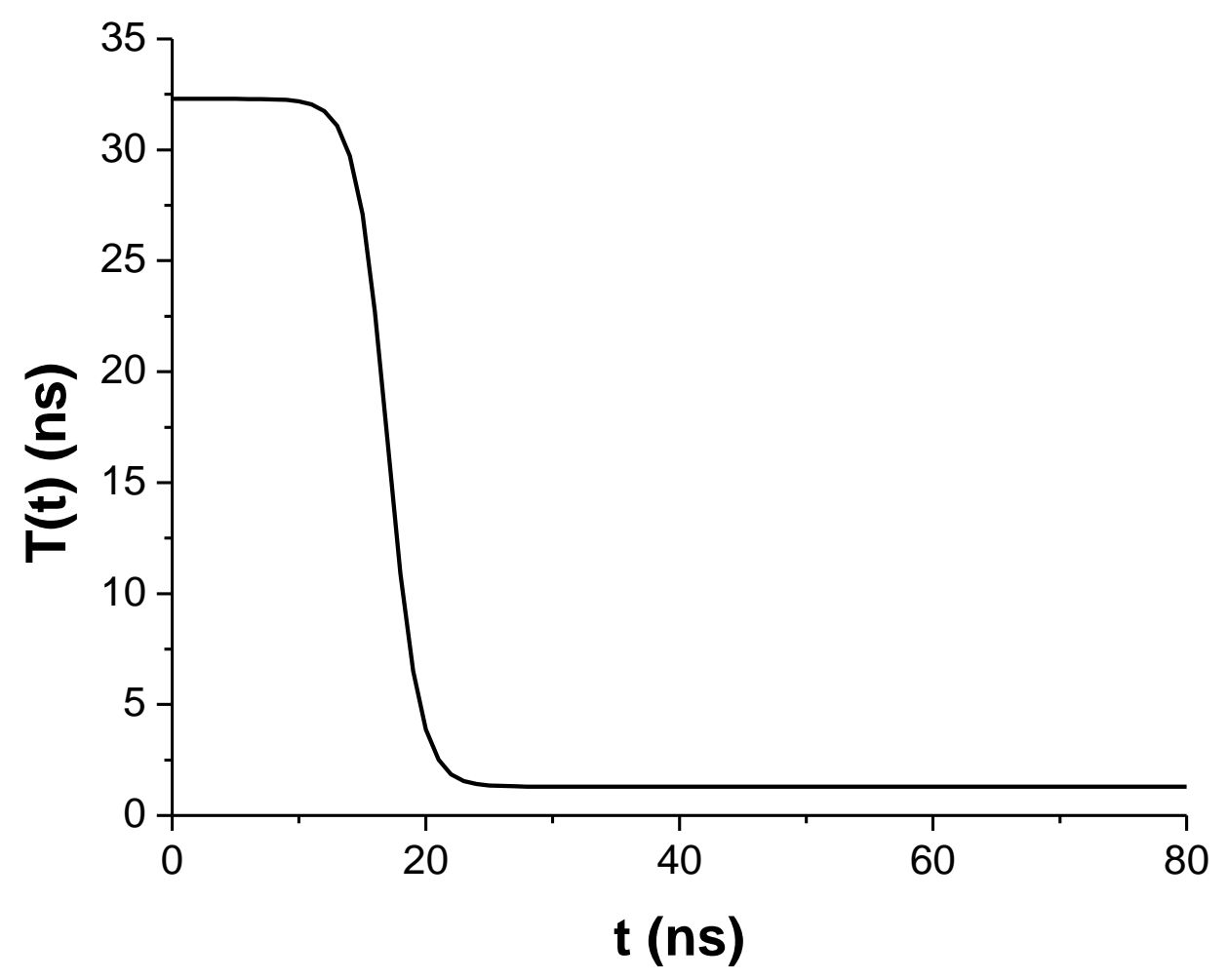


Figure 11

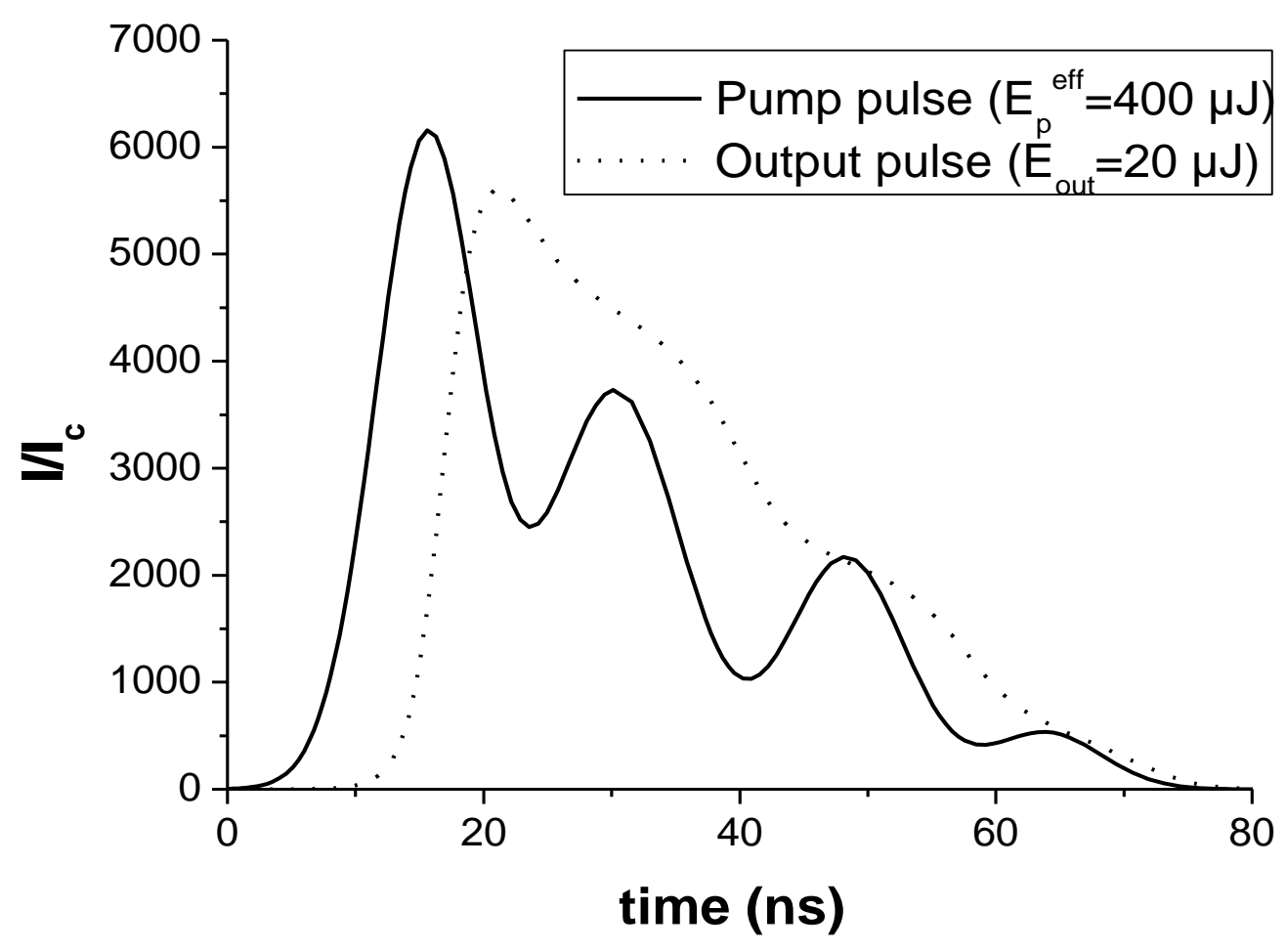

